\title{
Comparison between NAFLD fibrosis score and retinoic acid serum level in NAFLD
}

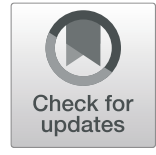

\author{
Ahmed Samir Allam ${ }^{1 *}$ D, Mohamed Magdy Salama ${ }^{1}$, Haytham Mohamed Nasser², \\ Walaa Ahmed Yousry Kabiel ${ }^{3}$ and Ehab H. Elsayed ${ }^{4}$
}

\begin{abstract}
Background: Non-alcoholic fatty liver disease (NAFLD) is described by the abnormal accumulation of fats in livers of individuals without significant alcohol intake. It includes a spectrum of diseases from simple steatosis to steatohepatitis (NASH) with fibrosis and cirrhosis. The prevalence of NAFLD is rising in association with increasing obesity worldwide. Retinoic acid (RA), a metabolite of vitamin A, mediates the functions of vitamin A required for growth and development. Also, RA has been shown to reduce adiposity not only in fat cells but also in the liver through increasing triglyceride hydrolysis and fat oxidation. This could put a future trial of preventing NASH and cirrhosis development by vitamin A supplementation. This work aimed to study the role of retinoic acid in NAFLD, whether it can differentiate simple steatosis from NASH and correlate the result with the NAFLD fibrosis score. It is a cross-sectional study done on 180 patients divided into three groups. Group 1 is composed of 80 patients with simple steatosis and normal ALT; group 2 is composed of 80 patients with NASH and high ALT in addition to group 3 with 20 healthy subjects served as a control group. All patients were proven to have fatty liver by ultrasonography. Serum RA was assayed by using enzyme-linked immunosorbent assay (ELISA) technique, and the NAFLD fibrosis score was calculated and compared with the retinoic acid level.

Result: Serum RA level was significantly decreased in the patient groups as compared to the controls; the lowest serum level was observed among the NASH group, followed by the steatosis group. NAFLD fibrosis score was calculated, and it was higher in the NASH group than in the steatosis group. Besides, there was a significant negative correlation between retinoic acid and NAFLD score among the patient groups.

Conclusion: Serum RA level was lower in patients with simple steatosis and NASH. RA had a high statistically significant difference in differentiation between the patient groups and the control group. The results were comparable to the NAFLD fibrosis score. Thus, retinoic acid could be used for diagnosis and accessing the degree of NAFLD.
\end{abstract}

Keywords: NAFLD, NASH, RA, ELISA, NAFLD fibrosis score

\section{Background}

Non-alcoholic fatty liver disease (NAFLD) is defined as the presence of hepatic steatosis in the absence of other causes of fat deposition in the liver. NAFLD includes simple steatosis, steatosis with various degrees of inflammation, and fibrosis, which is called non-alcoholic steatohepatitis (NASH). NAFLD is considered the most prevalent liver disease as a result of increased obesity incidence worldwide [1].

\footnotetext{
* Correspondence: ahm82allam@gmail.com

${ }^{1}$ Internal Medicine Department, Faculty of Medicine, Ain Shams University, Cairo 11211, Egypt

Full list of author information is available at the end of the article
}

Simple steatosis is a benign disease; however, NASH is considered to be a potentially health-threatening inflammation that may progress to cirrhosis, liver cell failure, and Hepatocellular carcinoma (HCC) [2]. Recently, there has been an increase in the mortality rate and the number of liver transplantations as a result of NASH complications. Non-alcoholic steatohepatitis (NASH) is now the second indication for liver transplantation in the USA [3].

At present, the gold standard technique for NASH diagnosis is a liver biopsy; nonetheless, it is an invasive procedure with possible serious complications and limitations [4]. NAFLD diagnosis requires the presence of 
hepatic steatosis either by imaging or histology in the absence of significant alcohol consumption [5].

Scoring systems using clinical and/or laboratory parameters to identify patients with NASH from the larger pool of NAFLD patients have been evaluated [6]. The most commonly used is the NAFLD fibrosis scoring system, which uses six commonly measured parameters. These include age, hyperglycemia, body mass index (BMI), platelet count, albumin level, and AST/ALT ratio. By using a low cut-off score, the NPV for excluding advanced fibrosis was around $88 \%$. When a high cut-off scores (0.676) was used, the PPV for predicting advanced fibrosis was almost 90\% [7].

Many serum markers were proposed for diagnosis of the NAFLD, but all are still not yet approved [8]. Retinoic acid (RA), a metabolite of vitamin A (retinol), mediates the functions of vitamin A required for growth and development through binding with the retinoic acid receptor (RAR) and retinoic acid X receptor (RXR), which then regulates the target gene expression [9].

Vitamin A is linked to NAFLD as the liver is the primary storage site of vitamin $\mathrm{A}$, and vitamin $\mathrm{A}$ has a role in the control of adipose tissue [10]. Vitamin A is stored in the quiescent hepatic stellate cells (qHSC), and these cells are responsible for fibrogenesis. When qHSC get activated to become profibrogenic $\mathrm{HSC}$, the activated HSC lose their vitamin A content. The retinoic acid role was proposed to effectively reduce adiposity in the liver and enhance hepatic fat catabolism [11].

\section{Aim of the work}

The present work aimed to study the clinical relevance of retinoic acid in patients with NAFLD and to detect its ability to differentiate simple steatosis from NASH and correlate its results with NAFLD fibrosis score.

\section{Methods}

It is a cross-sectional study. Subjects in this study were recruited from the in-patient ward and the outpatient clinic of the Internal Medicine Department of Ain Shams University Hospitals from the period of October 2016 to January 2018. Informed written consent was obtained from each participant before enrolment in the study. Moreover, the study was approved by the Research Ethics Committee of Ain Shams University.

The included subjects were classified into group 1 which is composed of 80 patients with simple steatosis proved by ultrasound to have fatty liver, with normal ALT, and group 2 which is composed of 80 patients with non-alcoholic steatohepatitis (NASH) proved by ultrasound to have fatty liver, with high ALT. All other causes of hepatitis, fibrosis, and cirrhosis such as viral hepatitis $\mathrm{B}$ and $\mathrm{C}$, alcoholic liver disease, drug-induced hepatitis, e.g., isoniazid and $\alpha$ methyldopa, drug-induced fatty liver, e.g., corticosteroids and amiodarone, metabolic liver diseases including Wilson disease, $\alpha-1$ antitrypsin and hemochromatosis, autoimmune hepatitis, primary biliary cirrhosis, and sclerosing cholangitis were excluded from the study. In addition to group 3, 20 age and sex-matched subjects, served as a healthy control group.

A full history was taken from all individuals included in this study with a thorough clinical examination with particular emphasis on anthropometric evaluation (measures of body weight and height) for calculation of body mass index. All participants in this study were subjected to routine laboratory hematology and chemistry assay including complete blood picture, liver function tests (albumin, INR, total and direct bilirubin, ALT, AST, and alkaline phosphatase), $\mathrm{HCV} \mathrm{Ab}$ and $\mathrm{HBsAg}$, kidney function tests (serum creatinine and blood urea), fasting blood sugar, HbA1C, lipid profile. Estimation of serum retinoic acid level throughout the enzyme-linked immunosorbent assay (ELISA) technique according to the manufacturer's instructions. Serum RA levels were measured using the commercial ELISA kit supplied by Life Science Company.

Abdominal ultrasonography was done for all subjects for diagnosis of non-alcoholic fatty liver and exclusion of other etiologies.

NAFLD fibrosis score was also calculated for all patients. It was calculated as per the following formula: $-1.675+0.037 \times$ age (years) $+0.094 \times$ body mass index $\left(\right.$ BMI, $\left.\mathrm{kg} / \mathrm{m}^{2}\right)+1.13 \times$ impaired fasting glucose/diabetes $($ yes $=1$, no $=0)+0.99 \times$ AST $/$ ALT ratio $-0.013 \times$ platelet $(\times 109 / \mathrm{L})-0.66 \times$ albumin $(\mathrm{g} / \mathrm{dL})$. The result was interpreted as low NFS $(<-1.445)$, indeterminate NFS ( -1.445 to 0.676$)$, and high NFS (>0.676) [12].

\section{Results}

This study included 180 patients who were divided into three groups: group 1, 80 patients with simple steatosis and normal ALT; group 2, 80 patients with NASH with high ALT; and group 3, 20 healthy subjects.

Table 1 showed that there was no significant difference between the studied groups regarding sex, age, and BMI.

Table 2 showed that there were no significant differences between the studied groups regarding AST, albumin, HbA1c, total cholesterol, and triglycerides. Retinoic acid level had high statistically significant difference among the studied groups, as the lowest levels were in NASH group, and the highest levels were in the control group. NAFLD score was higher in the NASH group than in the steatosis group, with a high statistically significant difference between the control and the patient groups.

Figure 1 showed that the retinoic acid level lowest levels were in NASH group, and the highest levels were in the control group with a high statistically significant difference among the studied groups. 
Table 1 Sex, age, and body mass index BMI among the studied groups

\begin{tabular}{|c|c|c|c|c|c|}
\hline Variables & Measures & Group 1 & Group 2 & Group 3 & $P$ \\
\hline \multirow{2}{*}{$\begin{array}{l}\text { Sex } \\
(n, \%)\end{array}$} & Male & $52(65.0 \%)$ & $50(62.5 \%)$ & $14(70.0 \%)$ & \multirow{2}{*}{$\begin{array}{l}b \\
0.902\end{array}$} \\
\hline & Female & 28 (35.0\%) & 30 (37.5\%) & $6(30.0 \%)$ & \\
\hline \multirow{2}{*}{$\begin{array}{l}\text { Age } \\
\text { (years) }\end{array}$} & Mean \pm SD & $53.5 \pm 6.3$ & $53.5 \pm 5.2$ & $52.5 \pm 6.3$ & \multirow{2}{*}{$\begin{array}{l}\text { a } \\
0.880\end{array}$} \\
\hline & Range & $40.0-76.0$ & $42.0-66.0$ & $40.0-61.0$ & \\
\hline \multirow{2}{*}{$\begin{array}{l}\mathrm{BMI} \\
\left(\mathrm{kg} / \mathrm{m}^{2}\right)\end{array}$} & Mean \pm SD & $30.9 \pm 2.2$ & $31.0 \pm 2.2$ & $29.0 \pm 1.2$ & \multirow{2}{*}{$\begin{array}{l}a \\
0.82\end{array}$} \\
\hline & Range & $25.9-36.3$ & $27.3-37.3$ & $26.7-34.3$ & \\
\hline
\end{tabular}

Group 1 simple steatosis and normal ALT, Group 2 NASH and high ALT, Group 3 control group, $B M I$ body mass index, $S D$ standard deviation

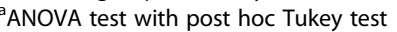

${ }^{\mathrm{b}} \mathrm{Chi}$ square test

Figure 2 showed that the NAFLD score was higher in the NASH group than the steatosis group, with a high statistically significant difference in the differentiation between NASH Group and the steatosis group.

Table 3 showed that there were significant negative correlations between $\mathrm{HbA1C}$, $\mathrm{TC}$, retinoic acid, and NAFLD score among the patient groups.

Table 4 showed that retinoic acid level showed a high statistically significant difference among the studied groups. NAFLD score had a high statistically significant difference in the differentiation between the NASH group and the steatosis group.

Table 5 showed that in the differentiation between group 1 and group 3 , retinoic acid value $\leq 120.0$ had high diagnostic accuracy, while NAFLD score $\geq-3.25$ had high sensitivity and PPV.
Table 6 showed that in differentiation between group 1 and group 2 , retinoic acid value $\leq 87.0$ had high sensitivity and negative predictive value, while NAFLD score $\geq-0.10$ had a high specificity and positive predictive value.

\section{Discussion}

At present, non-alcoholic fatty liver disease (NAFLD) is assumed to be the most common chronic liver disease. Retinoic acid (RA) is primarily involved in the regulation of lipid metabolism and hepatic steatosis [13].

Although some studies showed that RA supplementation might be a promising therapeutic approach in fatty liver disease, the clinical relevance of RA in the pathogenesis of hepatic steatosis and NAFLD has not been carefully investigated. So, the present study was designed to study the role of retinoic acid in the development and progression of NAFLD from simple steatosis to NASH and to compare the results with the NAFLD fibrosis score. After approval of the institutional review board, this study was conducted at the Internal Medicine Department, Ain Shams University Hospitals. Informed consent was obtained from all subjects enrolled in the study.

Our study obtained samples from 80 patients in group 1 (52 males and 28 females), 80 patients in group 2 (50 males and 30 females), and 20 control patients (14 males and 6 females). There were no significant differences between the studied groups regarding age and sex. Also, Liu et al. (2015) [14] showed that were no significant differences in age or female to male ratio between subjects in control, NAFLD, and NASH groups. On the other hand, Chaves et al. (2008) [15] showed that the mean

Table 2 AST, albumin, HbA1C, total cholesterol, and triglycerides among the studied groups

\begin{tabular}{|c|c|c|c|c|c|}
\hline Variables & Measures & Group 1 & Group 2 & Group 3 & P \\
\hline \multirow{2}{*}{$\begin{array}{l}\text { AST } \\
(\mathrm{IU} / \mathrm{L})\end{array}$} & Mean \pm SD & $27.1 \pm 13.1$ & $27.8 \pm 16.2$ & $26.2 \pm 3.4$ & \multirow[t]{2}{*}{0.944} \\
\hline & Range & $14.0-79.0$ & $11.0-75.0$ & $21.0-31.0$ & \\
\hline \multirow{2}{*}{$\begin{array}{l}\text { Albumin } \\
\text { (gm/dL) }\end{array}$} & Mean \pm SD & $3.7 \pm 0.4$ & $3.6 \pm 0.4$ & $4.2 \pm 0.4$ & \multirow[t]{2}{*}{0.935} \\
\hline & Range & $3.2-4.9$ & $3.4-4.3$ & $3.8-4.8$ & \\
\hline \multirow[t]{2}{*}{$\mathrm{HbA} 1 \mathrm{C}$} & Mean \pm SD & $5.9 \pm 1.1$ & $6.2 \pm 1.8$ & $4.9 \pm 0.95$ & \multirow[t]{2}{*}{0.044} \\
\hline & Range & $5.3-6.9$ & $5.7-7.8$ & $4.75-5.7$ & \\
\hline \multirow[t]{2}{*}{ Total cholesterol (mg/dl) } & Mean \pm SD & $196 \pm 36$ & $176 \pm 43$ & $180 \pm 42$ & \multirow[t]{2}{*}{0.079} \\
\hline & Range & $180-213$ & $216-263$ & $160-190$ & \\
\hline \multirow{2}{*}{$\begin{array}{l}\text { Triglycerides } \\
(\mathrm{mg} / \mathrm{dl})\end{array}$} & Mean \pm SD & $170 \pm 32$ & $186 \pm 44$ & $122 \pm 28$ & \multirow[t]{2}{*}{0.064} \\
\hline & Range & $140-210$ & $153-237$ & $110-150$ & \\
\hline \multirow[t]{2}{*}{ Retinoic Acid (ng/ml) } & Mean \pm SD & $110.1 \pm 39.9$ & $54.7 \pm 28.4$ & $190.9 \pm 37.2$ & \multirow[t]{2}{*}{$<0.001^{a}$} \\
\hline & Range & $68-225$ & 16-102 & $125-230$ & \\
\hline \multirow[t]{2}{*}{ NAFLD score } & Mean \pm SD & $-1.97 \pm 1.63$ & $0.68 \pm 1.18$ & $-2.83 \pm 0.57$ & \multirow[t]{2}{*}{$<0.001^{\mathrm{a}}$} \\
\hline & Range & -5.80 to -1.12 & $-2.27-6.12$ & -3.66 to -2.12 & \\
\hline
\end{tabular}

Group 1 simple steatosis and normal ALT, Group 2 NASH and high ALT, Group 3 control group, ALT alanine aminotransferase, AST aspartate aminotransferase ANOVA test with post hoc Tukey test

${ }^{a}$ Significant 


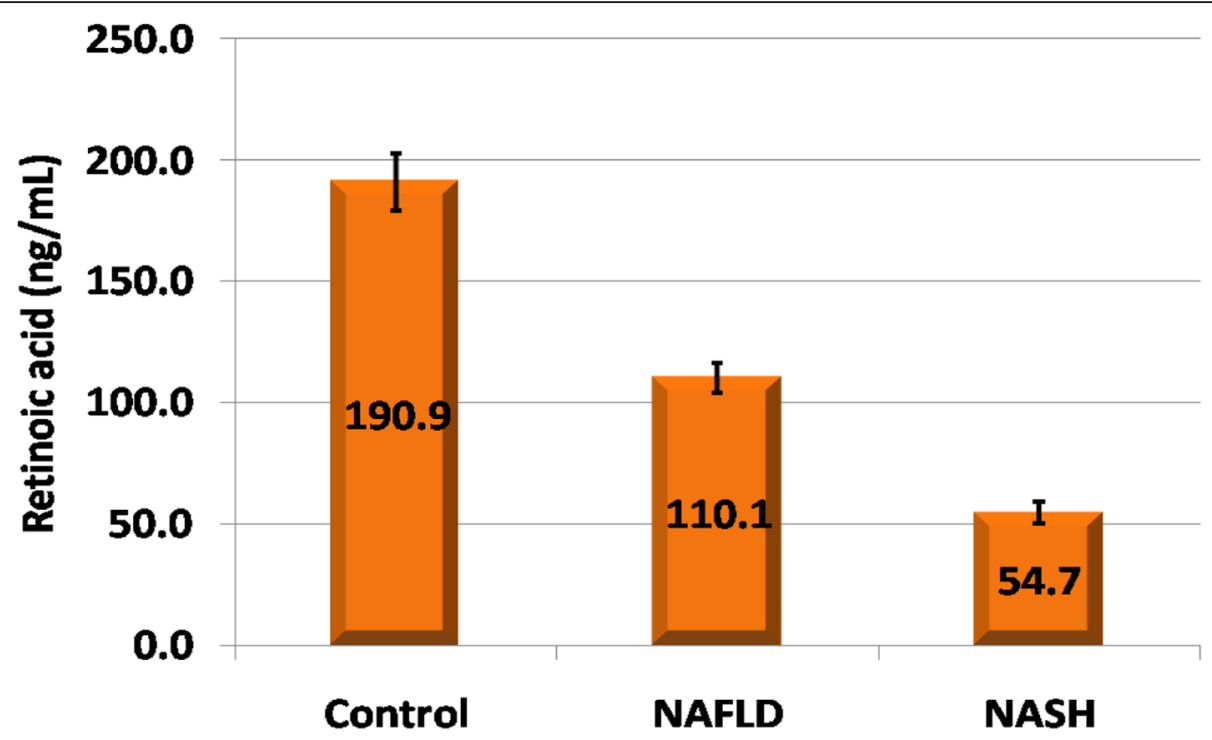

Fig. 1 Retinoic acid among the studied groups (normal level $9.3 \mathrm{ng} / \mathrm{ml}-300 \mathrm{ng} / \mathrm{ml}$ )

age was significantly higher within the NAFLD group with and without steatosis.

Also, there was no significant difference in BMI between the studied groups, and this in disagreement with the studies of Liu et al. (2015) ${ }^{(\mathbf{1 4})}$ and Bottella et al. (2011) [16] who showed significant difference in BMI between studied groups with higher BMI in NAFLD than the control groups; however, in their study, they selected patient group to be obese from the start. The high prevalence of obesity could explain this in Egyptian people; that is why both patients and controls were obese from the start with no significant statistical difference between them.

The present study showed that FBG had the lowest levels among the control group, followed by the steatosis group and had the highest levels in the NASH group, with no significant differences between the studied groups. Liu et al. (2015) [14] stated that the concentration of fasting plasma glucose in subjects with NAFLD and NASH was significantly higher than that in control subjects.

Miyazaki et al. 2012 [17] showed that BMI, ALT, and LDH were significantly higher in NAFLD patients than in the control group. Nutritional parameters, including total cholesterol, triglyceride, and fasting plasma glucose levels, were higher in the NAFLD patients than in the control group, although these differences were not statistically significant.

HbA1C was significantly higher in the patient groups than the control group with no significant difference between the steatosis group and the NASH group, but TG and TC showed no significant difference between studied groups.

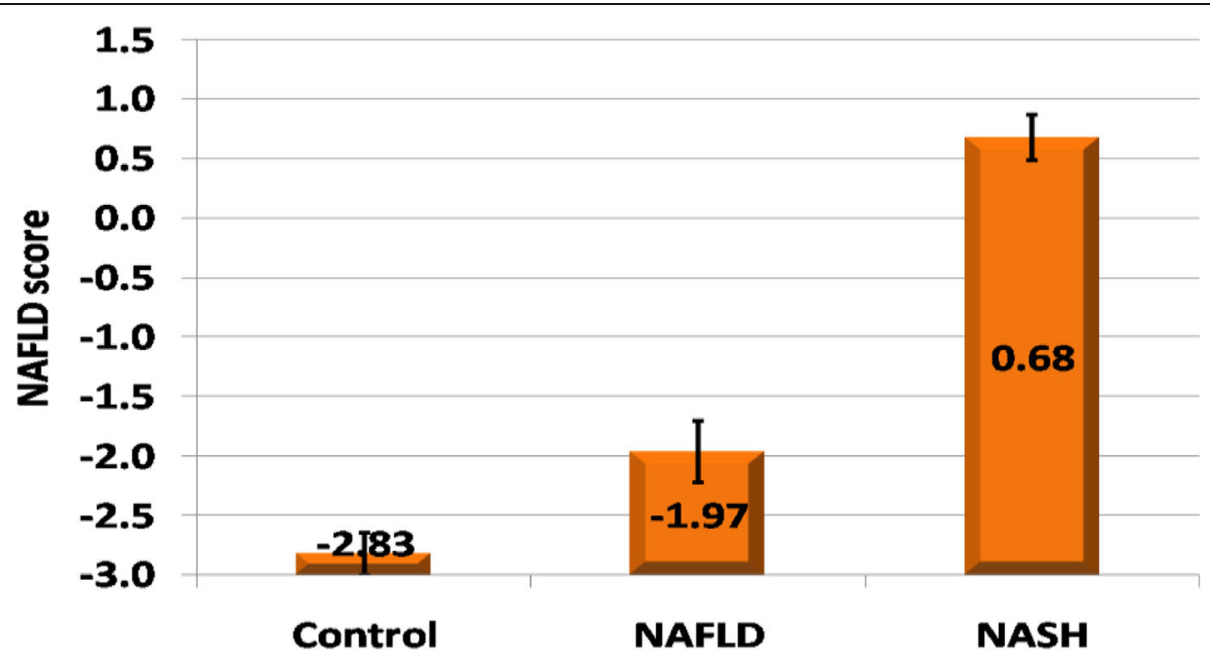

Fig. 2 NAFLD score among the studied groups 
Table 3 Correlation between retinoic acid and other variables among the studied groups

\begin{tabular}{|c|c|c|c|c|}
\hline Variables & Measures & Group 1 & Group 2 & Group 3 \\
\hline \multirow[t]{2}{*}{ Age } & $R$ & -0.023 & -0.135 & -0.084 \\
\hline & P & 0.890 & 0.735 & 0.817 \\
\hline \multirow[t]{2}{*}{ BMl } & $R$ & -0.178 & -0.087 & 0.387 \\
\hline & P & 0.271 & 0.598 & 0.270 \\
\hline \multirow[t]{2}{*}{ FBG } & $R$ & -0.213 & -0.171 & 0.203 \\
\hline & P & 0.187 & 0.293 & 0.575 \\
\hline \multirow[t]{2}{*}{ AST } & $R$ & -0.072 & -0.005 & 0.028 \\
\hline & P & 0.658 & 0.977 & 0.939 \\
\hline \multirow[t]{2}{*}{ PLT } & $R$ & 0.185 & 0.169 & -0.136 \\
\hline & $P$ & 0.874 & 0.298 & 0.708 \\
\hline \multirow[t]{2}{*}{ Albumin } & $R$ & 0.082 & 0.166 & 0.318 \\
\hline & $P$ & 0.613 & 0.306 & 0.371 \\
\hline \multirow[t]{2}{*}{ NAFLD score } & $R$ & -0.327 & -0.499 & 0.285 \\
\hline & $P$ & $0.039^{a}$ & $<0.001^{a}$ & 0.424 \\
\hline \multirow[t]{2}{*}{$\mathrm{HbA} 1 \mathrm{C}$} & $R$ & -0.427 & -0.627 & -0.072 \\
\hline & $P$ & $0.029^{a}$ & $0.019^{a}$ & 0.658 \\
\hline \multirow[t]{2}{*}{ TC (mg/dl) } & $R$ & -0.0 .528 & -0.0 .728 & 0.387 \\
\hline & P & $0.0 .015^{\mathrm{a}}$ & $0.0 .005^{\mathrm{a}}$ & 0.270 \\
\hline \multirow[t]{2}{*}{ T.G (mg) } & $R$ & -0.359 & -0.659 & -0.087 \\
\hline & P & $0.032^{a}$ & 0.244 & 0.598 \\
\hline
\end{tabular}

Group 1 simple steatosis and normal ALT, Group 2 NASH and high ALT, Group 3 control group, BMI body mass index, FBG fasting blood glucose, AST aspartate aminotransferase, PLT platelets, TC total cholesterol, TG triglycerides Pearson correlation, ${ }^{\mathrm{a}}$ Significant

Our study showed that serum retinoic acid level was significantly different among the studied groups, was lowest in the NASH group, followed by the steatosis group and highest in the control group and this was in accordance with Mourad et al. 2018 [18] and Pettinelli et al. 2018 [19].

However, other studies displayed no significant differences between control and NAFLD adults as Cengiz et al. 2010 [20] or even higher serum retinol in patients with NAFLD compared with a control group in Alkhouri et al. 2009 [21]. Senno et al. 2010 [11] found a positive correlation between serum retinol values and concentrations of AST and ALT in NAFLD. Serum retinol was the
Table 5 Diagnostic characteristics of retinoic acid and NAFLD score in differentiating group 1 from group 3

\begin{tabular}{lll}
\hline Characters & Value & $95 \% \mathrm{Cl}$ \\
\hline Retinoic acid $\leq 120.0$ & & \\
Sensitivity & $80.0 \%$ & $64.4-90.9 \%$ \\
Specificity & $76.3 \%$ & $69.2-100.0 \%$ \\
Diagnostic accuracy (DA) & $84.0 \%$ & $70.9-92.8 \%$ \\
Positive predictive value (PPV) & $74.2 \%$ & $89.1-100.0 \%$ \\
Negative predictive value (NPV) & $55.6 \%$ & $30.8-78.5 \%$ \\
Positive likelihood ratio (LR+) & $>100.0$ & $>100.0->100.0$ \\
Negative likelihood ratio (LR-) & 0.20 & $0.11-0.37$ \\
NAFLD score $\geq-3.25$ & & \\
Sensitivity & $85.0 \%$ & $70.2-94.3 \%$ \\
Specificity & $60.0 \%$ & $26.2-87.8 \%$ \\
Diagnostic accuracy (DA) & $80.0 \%$ & $66.3-90.0 \%$ \\
Positive predictive value (PPV) & $89.5 \%$ & $75.2-97.1 \%$ \\
Negative predictive value (NPV) & $50.0 \%$ & $21.1-78.9 \%$ \\
Positive likelihood ratio (LR+) & 2.13 & $0.98-4.59$ \\
Negative likelihood ratio (LR-) & 0.25 & $0.10-0.61$ \\
\hline Cl confide in
\end{tabular}

Cl confidence interval, Group 1 simple steatosis and normal ALT, Group 2 NASH and high ALT, Group 3 control group

only biochemical variable that could predict AST and ALT concentrations in these patients, pointing to retinol as a potential marker for liver damage.

NAFLD score was higher in the NASH group than the steatosis group, with a high statistically significant difference in the differentiation between NASH group and the steatosis group.

The correlation analysis revealed a negative correlation between retinoic acid and NAFLD score among the steatosis group and the NASH group. The receiver operating characteristics (ROC) curve was used to define the best cutoff value of serum retinoic acid level. Retinoic acid had a significantly high statistical difference in the differentiation between the study groups similar to that of NAFLD fibrosis score.

Some limitations need to be discussed in the present study. First, more randomized controlled clinical trials in human subjects are required to confirm the fundamental role of low RA concentrations in NAFLD and to correlate the results with a liver biopsy; besides, the possible

Table 4 Diagnostic performance of retinoic acid and NAFLD score in differentiation between the studied groups

\begin{tabular}{lllllll}
\hline Group & Variable & AUC & SE & $P$ & $95 \%$ Cl & Cut-off \\
\hline Group 1 from group 3 & Retinoic & 0.920 & 0.038 & $<0.001^{\mathrm{a}}$ & $0.845-0.995$ & $\leq 120.0$ \\
& Score & 0.785 & 0.063 & $0.006^{\mathrm{a}}$ & $0.662-0.908$ & $\geq-3.25$ \\
Group 2 from group 1 & Retinoic & 0.912 & 0.030 & $<0.001^{\mathrm{a}}$ & $0.852-0.971$ & $\leq 87.0$ \\
& Score & 0.942 & 0.025 & $<0.001^{\mathrm{a}}$ & $0.893-0.991$ & $\geq-0.10$ \\
\hline
\end{tabular}

AUC area under curve, SE standard error, Cl confidence interval, Group 1 simple steatosis and normal ALT, Group 2 NASH and high ALT, Group 3 control group ${ }^{\text {a }}$ Significant 
Table 6 Diagnostic characteristics of retinoic acid and NAFLD score in differentiating group 1 from group 2

\begin{tabular}{lll}
\hline Characters & Value & $95 \% \mathrm{Cl}$ \\
\hline Retinoic acid $\leq 87.0$ & & \\
Sensitivity & $82.5 \%$ & $67.2-92.7 \%$ \\
Specificity & $77.5 \%$ & $61.5-89.2 \%$ \\
Diagnostic accuracy (DA) & $80.0 \%$ & $69.6-88.1 \%$ \\
Positive predictive value (PPV) & $78.6 \%$ & $63.2-89.7 \%$ \\
Negative predictive value (NPV) & $81.6 \%$ & $65.7-92.3 \%$ \\
Positive likelihood ratio (LR+) & 3.67 & $2.03-6.63$ \\
Negative likelihood ratio (LR-) & 0.23 & $0.11-0.45$ \\
NAFLD score $\geq-0.10$ & & \\
Sensitivity & $80.0 \%$ & $64.4-90.9 \%$ \\
Specificity & $97.5 \%$ & $86.8-99.9 \%$ \\
Diagnostic accuracy (DA) & $88.8 \%$ & $79.7-94.7 \%$ \\
Positive predictive value (PPV) & $97.0 \%$ & $84.2-99.9 \%$ \\
Negative predictive value (NPV) & $83.0 \%$ & $69.2-92.4 \%$ \\
Positive likelihood ratio (LR+) & 32.00 & $4.59-223.01$ \\
Negative likelihood ratio (LR-) & 0.21 & $0.11-0.38$ \\
\hline C confidence & & \\
\hline
\end{tabular}

$\mathrm{Cl}$ confidence interval, Group 1 simple steatosis and normal ALT, Group 2 NASH and high ALT, Group 3 control group

preventive or therapeutic advantage of RA needs to be evaluated to confirm its benefits and safety.

Furthermore, in diagnosing NAFLD in the studied groups, most of the previous studies used ultrasound only, which is limited by its inability to differentiate mild steatosis from fibrosis and to accurately quantify fatty infiltration; besides, it is an operator dependent. Others used a liver biopsy, which is an invasive method with possible serious complications. Nevertheless, in our study, we used, in addition to ultrasound, NAFLD fibrosis score, which is one of the accurate scores in diagnosing and differentiation simple steatosis from NASH and compared its results with RA serum levels.

Magnetic resonance elastography (MRE) is a noninvasive medical imaging technique that measures liver stiffness can also be used; however, the issue of the cost and the availability of the MRI needs to be discussed. This may open new insights on the use of serum retinoic acid in NAFLD as regards the cost per test, and being a single biomarker could be less costly than variable laboratory parameters already used right now or imaging techniques as MRE.

\section{Conclusion}

The present study showed that serum RA was lower in patients with simple steatosis and NASH with high statistical significant differences in differentiation between the patient groups and the control group. NAFLD fibrosis score had high sensitivity in differentiating the NAFLD groups from the control group and in differentiating the steatosis group from the NASH group. Additionally, there were significant negative correlations between retinoic acid and NAFLD score among steatosis and NASH groups. Serum RA concentration is lowest in the NASH group. Consequently, Retinoic Acid can be used as a single noninvasive tool for diagnosis and assessing the degree of NAFLD and possibly differentiating simple steatosis from NASH.

\section{Abbreviations}

ALT : Alanine aminotransferase; ANOVA: Analysis of variance; AST: Aspartate aminotransferase; AUC: Area under the curve; BMl: Body mass index; CBC: Complete blood count; Cl: Confidence interval; DA: Diagnostic accuracy; ELISA: Enzyme-linked immunosorbent assay; FBG: Fasting blood glucose; Hb: Hemoglobin; HBV: Hepatitis B virus; HCC: Hepatocellular carcinoma; HCV: Hepatitis C virus; HSC: Hepatic stellate cells; INR: International normalized ratio; K: Potassium; LR+: Positive likelihood ratio; LR-: Negative likelihood ratio; MR: Magnetic resonance elastography; Na: Sodium; NAFLD: Non-alcoholic fatty liver disease; NASH: Non-alcoholic steatohepatitis:; NFS: NAFLD fibrosis score; NPV: Negative predictive value; PLT: Platelets; PPV: Positive predictive value; qHSC: quiescent hepatic stellate cells; RA: Retinoic acid; RAR: Retinoic acid receptor; ROC-curve: Receiver operating characteristic curve; RXR: Retinoic acid X receptor; SD: Standard deviation; TC: Total cholesterol; TG: Triglycerides

\section{Acknowledgements}

Not applicable

\section{Authors' contributions}

ASA and MMS conceived and planned the experiments. WAK contributed to sample preparation. HMN performed the ultrasound to the patients. EE contributed to final manuscript editing and revision. All authors provided critical feedback and helped shape the research, analysis and manuscript. All authors have read and approved the manuscript

\section{Funding}

The funders had no role in study design, data collection and analysis, decision to publish, or preparation of the manuscript.

\section{Availability of data and materials}

The authors confirm that the data supporting the findings of this study are available within the article.

\section{Ethics approval and consent to participate}

All procedures performed in this study were in accordance with the ethical standards of the Ain Shams University Research Committee and with the 1964 Helsinki Declaration and its later amendments.

Ethics committee's reference number: 000017585.

Address: Faculty of Medicine, Ain Shams University, Cairo 11211, Egypt. Informed written consent was obtained from each participant before enrolment in the study.

\section{Consent for publication}

Informed consent to publish patient's data was signed by all participants prior to the beginning of the research.

\section{Competing interests}

The authors declare that they have no competing interest.

\section{Author details}

${ }^{1}$ Internal Medicine Department, Faculty of Medicine, Ain Shams University, Cairo 11211, Egypt. ${ }^{2}$ Radiology Department, Faculty of Medicine, Ain Shams University, Cairo, Egypt. ${ }^{3}$ Clinical Pathology Department, Faculty of Medicine, Ain Shams University, Cairo 11211, Egypt. ${ }^{4}$ Tropical Medicine Department, National Research Center, Cairo 11211, Egypt. 
Received: 23 September 2019 Accepted: 12 December 2019

Published online: 14 January 2020

\section{References}

1. Hazlehurst JM, and Tomlinson JW (2013)Non-alcoholic fatty liver disease in common endocrine disorders 169:R27-37. doi: https://doi.org/10.1530/EJE-13-0296

2. Nascimbeni F, Pais R, Bellentani $S$ et al (2013) From NAFLD in clinical practice to answers from guidelines. J Hepatol 59:859-871

3. Zhu JZ, Dai YN, Wang YM et al (2015) Prevalence of nonalcoholic fatty liver disease and economy. Dig Dis Sci 60:3194-3202

4. Bell LN, Theodorakis JL, Vuppalanchi R et al (2010) Serum proteomics and biomarker discovery across the spectrum of nonalcoholic fatty liver disease. Hepatology 51:111-120

5. Chalasani N, Younossi Z, Lavine JE et al (2012) The diagnosis and management of non-alcoholic fatty liver disease: practice guideline by the American Association for the Study of Liver Diseases, American College of Gastroenterology, and the Amer-ican Gastroenterological Association. Hepatology 55:2005-2023

6. Ratziu V, Giral P, Charlotte F et al (2000) Liver fibrosis in overweight patients. Gastroenterology 118:1117-1123

7. Angulo P, Hui JM, Marchesini G et al (2007) The NAFLD fibrosis score: a noninvasive system that identifies liver fibrosis in patients with NAFLD. Hepatology 45:846-854

8. Machado MV, Cortex-Pinto H (2013) Noninvasive diagnosis of non-alcoholic fatty liver disease: A critical appraisal. J Hepatol 58:1007-1019

9. Kam RK, Deng Y, Chen Y. et al. (2012) Retinoic acid synthesis and functions in early embyronic developement Cell Biosci 2: 11.

10. Mondul A, Mancina RM, Merlo A et al (2015) PNPLA3 I148M variant influences circulating retinol in adults with nonalcoholic fatty liver disease or obesity. J Nutr 145:1687-1691

11. Senoo H, Yoshikawa K, Morii M et al (2010) Hepatic stellate cell (vitamin Astor-ing cell) and it's relative - past, present and future. Cell Biol Int 34 1247-1272

12. Rinella M.(2015)Non-alcoholic fatty liver disease: a systematic review313: 2263-73. doi: https://doi.org/10.1001/jama.2015.5370.

13. Bonet ML, Ribot J, Palou A (2012) Lipid metabolism in mammalian tissues and its control by retinoic acid. Biochim Biophys Acta 1821:177-189

14. Liu Y, Chen H, Wang J et al (2015) Association of serum retinoic acid with hepatic steatosis and liver injury in non-alcoholic fatty liver disease. Am J Clin Nutr 102:130-137

15. Chaves G, Pereira SE, Saboya CJ et al (2008) Non-alcoholic Fatty Liver Disease and Its Relationship with the Nutritional Status of Vitamin A in Individuals with Class III Obesity. Obes Surg 18:378. https://doi.org/10.1007/ 11695-007-9361-2

16. Botella-Carretero Jl, Balsa JA, Vázquez C et al (2010) Retinol and alphatocopherol in morbid obesity and nonalcoholic fatty liver disease. Obes Surg 20:69-76

17. Miyazaki M, Kato M, Tanaka K et al (2012) Increased hepatic expression of dipeptidyl peptidase-4 in non-alcoholic fatty liver disease and its association with insulin resistance and glucose metabolism. Mol Med Rep 5:729-733

18. Mourad F, El Sayeda Z, Showmana H, Abo RM, Mohamed N (2018) The role of serum retinol in nonalcoholic fatty liver disease. Sci J Al-Azhar Med Fac Girls 2:115-122

19. Pettinelli P, Arendt BM, Teterina A, McGilvray I, Comelli EM, Fung SK, Fischer SE, Allard JP (2018) Altered hepatic genes related to retinol metabolism and plasma retinol in patients with non-alcoholic fatty liver disease. PLoS One. 13:e0205747

20. Cengiz C, Ardicoglu Y, Bulut S, Boyacioglu S (2010) Serum retinol-binding protein 4 in patients with nonalcoholic fatty liver disease: does it have a significant impact on pathogenesis? Eur J Gastroenterol Hepatol 22:813-819

21. Alkhouri N, Lopez R, Berk M, Feldstein A (2009) Serum retinol-binding protein 4 levels in patients with nonalcoholic fatty liver disease. J Clin Gastroenterol 43:985-989

\section{Publisher's Note}

Springer Nature remains neutral with regard to jurisdictional claims in published maps and institutional affiliations.

\section{Submit your manuscript to a SpringerOpen ${ }^{\circ}$ journal and benefit from:}

- Convenient online submission

- Rigorous peer review

- Open access: articles freely available online

- High visibility within the field

- Retaining the copyright to your article

Submit your next manuscript at $\boldsymbol{\nabla}$ springeropen.com 